\title{
Uncertainty in Tractography via Tract Confidence Regions
}

\author{
Colin J. Brown, Brian G. Booth, and Ghassan Hamarneh
}

\begin{abstract}
Tractography allows us to explore white matter connectivity in diffusion MR images of the brain. However, noise, artifacts and limited resolution introduce uncertainty into the results. We propose a statistical model that allows us to quantify and visualize the uncertainty of a neuronal pathway between any two fixed anatomical regions. Given a sample set of tract curves obtained via tractography, we use our statistical model to define a confidence region that exposes the location and magnitude of tract uncertainty. The approach is validated on both synthetic and real diffusion MR data and is shown to highlight uncertain regions that occur due to noise, fiber crossings, or pathology.
\end{abstract}

\section{Introduction}

Brain tractography is the process of discovering neuronal pathways in diffusion MR (dMR) images of the brain. A variety of methods exist to discover tracts, but with what certainty these tracts correspond to underlying anatomical fibers is often difficult to determine. Diffusion MR images are often of low resolution, noisy, and may contain artifacts caused by patient movement or the scanning process itself [14]. These problems can cause tractography algorithms to output false positives or tracts which stray from anatomical fibers. Kissing and crossing fibers can add further uncertainty to the estimated trajectory of neuronal fiber tracts. Thus, there is consid-

Colin J. Brown

Simon Fraser University, Burnaby, BC, Canada, e-mail: c jbrowne sfu.ca

Brian G. Booth

Simon Fraser University, Burnaby, BC, Canada, e-mail: bgb2 @ s fu . ca

Ghassan Hamarneh

Simon Fraser University, Burnaby, BC, Canada, e-mail: hamarneh@sfu.ca 
erable motivation to determine the confidence in the trajectory of a tractography result $[5,8]$. In other words, given two brain regions of interest, $A$ and $B$ in the image space $\Omega$, assumed to be connected, there is a need for a method to determine the confidence in the trajectory of the connecting pathway before proceeding to subsequent quantification or visualization tasks for any clinical application (e.g. image-guided neurosurgery $[6,18])$.

Previous works have attempted to address the problem of uncertainty in tractography in a variety of ways. One approach is to calculate the probability that two regions are connected. Given a fiber orientation distribution function (fODF) at each voxel, it is possible to define the marginal probability of a tract connecting $A$ and $B$ [1]. However, given the large domain of paths that connect $A$ and $B$, computing that marginal probability is often infeasible. Instead, one can sample tracts from $A$ using probabilistic tractography, and examine the frequency with which they pass through $B[1,11]$. While this method exposes the certainty of connectivity between two points, it does not directly provide insight into the range of possible path trajectories in uncertain regions. Alternatively, one can calculate the probability of a connection by taking the product of the individual conditional probabilities along a tract from $A$ to $B$ [11]. While this path probability may be a useful measure to compute, it is sensitive to noise and gives no clues about possible spatial variability in path trajectory. In particular, using this measure alone provides no way of discerning if other, similarly likely, paths exist. More recently, Brown et al. presented the k-confidence measure that relates tract confidence to the variability in the path trajectories of the $k$ most likely paths between $A$ and $B$ [3]. While this approach directly measures path variability, it only reports a single number and does not convey the location or nature of discovered uncertainty.

There have also been studies to investigate the accuracy and reliability of tractography algorithms by comparing results to tracts delineated by tracers administered to subjects in vitro [10, 4]. Unfortunately, these kinds of studies can only be performed post-mortem. The recently presented Tractometer test suite is also an attempt to reduce the difficulty in evaluating tractography methods [8]. While important, Tractometer only gives general reliability scores and not specific information about the uncertainty of tract trajectories in a novel image.

We propose a method for computing and visualizing a statistical confidence region for the distribution of likely tract curves between two regions in the brain. Given any tractography algorithm capable of discovering multiple candidate tracts between two regions $[1,3,11,13]$, we compute a sample set of tract curves. We use this sample set to estimate a distribution over the locations of the tracts, and we assume the mean of this distribution is the most likely location of the underlying anatomical tracts. We then compute a volumetric region that, for a given confidence level, will contain the mean (or "true") tract. This confidence region is wide along dimensions with high uncertainty and is compact or narrow along dimensions where the estimate of the true mean is more certain. If there is truly a single (nonbifurcated), clear connection between brain regions, we expect its tract distribution to be strongly peaked around one path, resulting in a narrow confidence region around the mean. If, instead, the regions are not connected or the image quality is 
too poor, then no single path should be highly favourable and the tract distribution would lead to a wide confidence region. This reasoning motivates our contribution: a method to compute tract confidence and visualize it in a way that reveals regions of uncertainty in discovered pathways.

\section{Method}

The proposed method proceeds in two steps. First, a sample set of tract curves, obtained via tractography $[1,3,11,13]$, is used to compute a statistical confidence region within which we can say, with a certain confidence, the mean tract lies. Once we have this high-dimensional confidence region, we proceed to map said region into the image space to enable inference about the connectivity and trajectory of neuronal fibers between the two regions.

\subsection{Path Confidence Regions}

Given a source and target region, $A$ and $B$, we first generate sample tract curves connecting $A$ and $B$. Formally, we represent each sample curve as a sequence of $3 \mathrm{D}$ points. When sampled, a tract curve may consist of a varying number of points depending on its total Euclidean length and the method of delineation. We therefore resample each curve to have exactly $n$ equally spaced points and represent it as a single $3 n$-dimensional observation (where the first three elements correspond to the three coordinates of the first point and so on). Each tract, $x^{i}$, in a sample of $K$ tracts, $i=1 \cdots K$, is denoted $x^{i}=\left(x_{0}^{i}, x_{1}^{i}, x_{2}^{i}, \cdots, x_{3 \rho}^{i}, x_{3 \rho+1}^{i}, x_{3 \rho+2}^{i}, \cdots, x_{3 n-1}^{i}\right)$. Note that each value of $\rho \in\{0, \cdots, n-1\}$ indexes a unique 3D point, denoted $x^{i}(\rho)=$ $\left(x_{3 \rho}^{i}, x_{3 \rho+1}^{i}, x_{3 \rho+2}^{i}\right)$, in a given tract.

The tract curve distribution to sample from is unknown but, for simplicity, we assume it to be a multivariate normal distribution. Given a sample set of tracts, one can calculate the mean tract and tract covariance matrix as

$$
\bar{x}_{j}=\frac{1}{K} \sum_{i=1}^{K} x_{j}^{i} \quad \text { (1) } \quad \Sigma_{j k}=\frac{1}{K} \sum_{i=1}^{K}\left(x_{j}^{i}-\bar{x}_{j}\right)\left(x_{k}^{i}-\bar{x}_{k}\right) .
$$

This statistical model, similar to that presented in [7], assumes our sample set is generated using unbiased sampling techniques. This assumption holds for probabilistic tractography algorithms $[1,11]$ and bootstrap tractography techniques [13], but not for $k$ optimal path tractography [3]. In the $k$ optimal path tractography algorithm, each of the $K$ most optimal paths between $A$ and $B$ are discovered exactly

once. Nevertheless, each path is associated with a path probability, $p\left(x^{i}\right)$, that we use as an estimate of sampling frequency. Accordingly, the mean and co-variance of the path distribution are instead estimated by the weighted mean and weighted co-variance of the $k$ optimal paths, computed as 


$$
\begin{gathered}
\bar{x}_{j}=\frac{1}{K \sum_{i=1}^{K} p\left(x^{i}\right)} \sum_{i=1}^{K} p\left(x^{i}\right) x_{j}^{i} \\
\Sigma_{j k}=\frac{\sum_{i=1}^{K} p\left(x^{i}\right)}{\left(\sum_{i=1}^{K} p\left(x^{i}\right)\right)^{2}-\sum_{i=1}^{K} p\left(x^{i}\right)^{2}} \sum_{i=1}^{K} p\left(x^{i}\right)\left(x_{j}^{i}-\bar{x}_{j}\right)\left(x_{k}^{i}-\bar{x}_{k}\right) .
\end{gathered}
$$

Given the above statistical models, the confidence region for the mean tract, with confidence level $100(1-\alpha) \%$, is defined [9] by the inequality,

$$
\frac{K(K-3 n)}{(K-1) 3 n}(\mu-\bar{x})^{T} \Sigma^{-1}(\mu-\bar{x}) \leq F_{3 n, K-3 n}(1-\alpha)
$$

where $F$ is the $F$-distribution and $\mu \in \mathbb{R}^{3 n}$. The confidence region of $\mu$ therefore defines a volume in $\mathbb{R}^{3 n}$.

\subsection{Confidence Region Visualization}

In order to visualize the confidence region in $3 \mathrm{D}$, we need to find which voxels in the image space $\Omega$ are traversed by tract curves, $\mu$, that satisfy (5). Clearly, testing every possible path between $A$ and $B$ for membership in the confidence region is infeasible. Instead, for each voxel $v \in \Omega$, we check whether it is feasible for a tract to pass through $v$ and satisfy (5). This leads to the following feasibility problem

$$
\begin{aligned}
& \min _{\mu, \rho} 0 \\
& \text { subject to } \frac{K(K-3 n)}{(K-1) 3 n}(\mu-\bar{x})^{T} \Sigma^{-1}(\mu-\bar{x}) \leq F_{3 n, K-3 n}(1-\alpha) \\
& \text { and } \mu(\rho)=v,
\end{aligned}
$$

in which we search for tracts, $\mu$, that satisfy (5) and pass through $v$ at some index $\rho$ (recall that $\mu(\rho)$ denotes any indexed 3D point in tract $\mu$ ). Note that the minimization of a constant $(\min 0)$ is standard notation in feasibility problems since we only need to ensure that the constraints are met [2, p. 258]. We use Lagrangian relaxation to convert this feasibility problem into a minimization problem [17, p. 27], for each $v$,

$$
\min _{\mu, \rho} \lambda\left[\frac{K(K-3 n)}{(K-1) 3 n}(\mu-\bar{x})^{T} \Sigma^{-1}(\mu-\bar{x})-F_{3 n, K-3 n}(1-\alpha)\right]
$$

subject to $\mu(\rho)=v$.

The Lagrange multiplier $\lambda$ and $F$-value $F_{3 n, K-3 n}(1-\alpha)$ are constant with respect to $\mu$ and $\rho$ and can be removed from the minimization without affecting the optimal 
solution. While the objective function in (7) is convex, the restriction, $\mu(p)=v$, is not. In order to achieve convexity, the minimization over $\rho$ is decoupled. We solve (7) by first fixing a value of $\rho$. This leads to the problem of choosing which 3D point in tract $\mu$ to anchor at voxel $v$. Intuitively, we select the index of the nearest point to $v$ on the sample mean tract, $\bar{x}\left(\rho^{*}\right)$, where nearest is defined using the Mahalanobis distance [15]. Formally, this leads to the optimization problem,

$$
\rho^{*}=\arg \min _{\rho} \sqrt{(v-\bar{x}(\rho))^{T} \Sigma_{\alpha(\rho), \alpha(\rho)}^{-1}(v-\bar{x}(\rho))},
$$

where $3 \rho \leq \alpha(\rho) \leq 3 \rho+2$ defines the sub-matrix $\Sigma_{\alpha(\rho), \alpha(\rho)}^{-1}$ of the inverse sample covariance matrix, $\Sigma^{-1}$. This minimization is performed via brute force as our tract length, $n$, is sufficiently small. ( $n$ was set to 150 in all of our experiments). Thus, given $v, \rho^{*}$ is uniquely selected and the minimization problem in (7) becomes,

$$
\begin{aligned}
& z(v)=\min _{\mu}\left[\frac{K(K-3 n)}{(K-1) 3 n}(\mu-\bar{x})^{T} \Sigma^{-1}(\mu-\bar{x})\right] \\
& \text { subject to } \mu\left(\rho^{*}\right)=v \text {. }
\end{aligned}
$$

This convex optimization can be interpreted as finding the minimum $F$-statistic at each voxel and the minimum is computed using interior point optimization [12]. If there exists a feasible solution, $\mu$, such that $z(v) \leq F_{3 n, K-3 n}(1-\alpha)$, then (5) is satisfied and $v$ is inside the confidence region. Otherwise, $v$ must be outside the confidence region. Minimizing (9) over every $v \in \mathscr{I}$ generates a volumetric confidence region,

$$
\mathscr{C}(\alpha)=\left\{v ; \forall v \in \mathscr{I} \text { s.t. } z(v) \leq F_{3 n, K-3 n}(1-\alpha)\right\} .
$$

\section{Results}

In this section we demonstrate both the correctness of tract confidence regions and their applicability to clinical data. We begin by examining tract confidence regions for two sets of diffusion MR phantoms, one with an isolated section containing increasing levels of noise and the other with a section of simulated crossing fibers at varying angles. Next, an experiment on brain diffusion MR images of healthy subjects is presented that tests the inter-subject consistency of our method and demonstrates the correlation between tract crossing regions and confidence region thickness. Finally, we examine whether tract confidence regions can highlight abnormalities in dMR images of human brains with Multiple Sclerosis (MS) and brain tumors.

Since the $F$ distribution depends on the number of tract curves, $K$, and the number of points per curve, $n$, the confidence region size is affected by these parameters. As expected, the confidence region grows with smaller $K$ and larger $n$. For all subsequent tests, we fix $K=500, n=150$ and $\alpha=0.01$ so that confidence region sizes are 


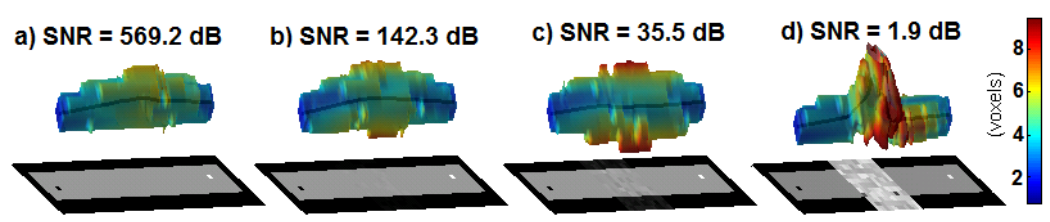

Fig. 1 Four dMR phantom images of a single tract with a narrow band of increasing levels of noise. Tract curves are sampled between end points and tract confidence regions are plotted above a representative fractional anisotropy (FA) slice of each phantom. Color denotes the distance from the weighted sample mean tract (black) at each point on the surface (i.e. bulges become redder). Note the increasing bulge size with reduced SNR.

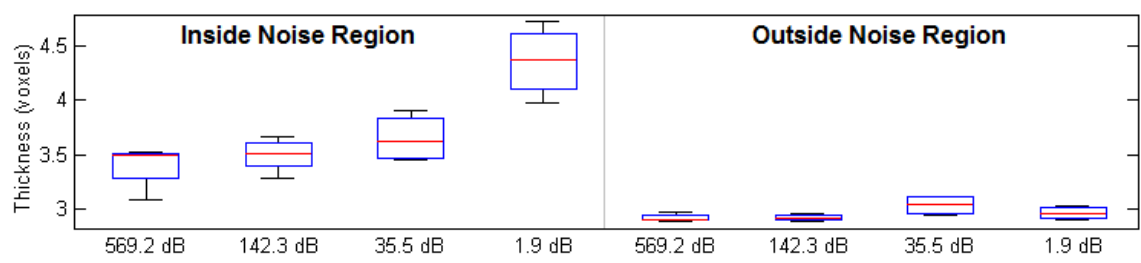

Fig. 2 Thicknesses of confidence regions, inside and outside of the noisy region, for each noise level in Fig. 1. Plot summarizes four realizations of noise at each level. Thickness is calculated as the average distance from the mean tract. Note that as noise increases, we obtain a thicker confidence region, implying higher tract uncertainty.

comparable across tests. Note that while the absolute thickness of a confidence region changes with $K$ and $n$, the relative bulges around regions of uncertainty should be consistent and relatively independent of specific parameter settings.

In the first experiment (Fig. 1), we generate a simple linear fiber bundle phantom with uniform diffusion tensors pointing along the bundle. Increasing levels of noise are added to a narrow (10-voxel wide) band, reducing the signal to noise ratio (SNR). We hypothesize that more noise will lead to wider confidence regions. We compute and visualize our confidence regions for $K$ sampled tracts (using k-optimal path tractography) between points on either end of the phantom. As expected, the noise causes uncertainty in the trajectory of the connection as evidenced by the bulging in the resulting confidence regions (Fig. 2).

The second phantom experiment is set up similarly but instead of noise, the narrow band contains a region of simulated crossing fibers. A tensor is added to each voxel of this band which deviates from the original primary tensor direction at increasing angles (Fig. 3). At larger angles, the primary tensor directions are nearly orthogonal, and thus there is a high degree of uncertainty about the most likely fiber trajectory. At smaller angles, the tensors nearly agree and so there is less uncertainty. As we expect, the tract confidence regions become thicker across the affected band as the angle becomes closer to $90^{\circ}$.

Next, our method is validated on 12 images from the John Hopkins MILBA MRI In Vivo Human Database [16]. Tracts are sampled along a fiber in the corpus callosum (CC) that crosses the corticospinal tract. We hypothesize that this crossing region causes greater uncertainty to the tractography algorithm and should result in 


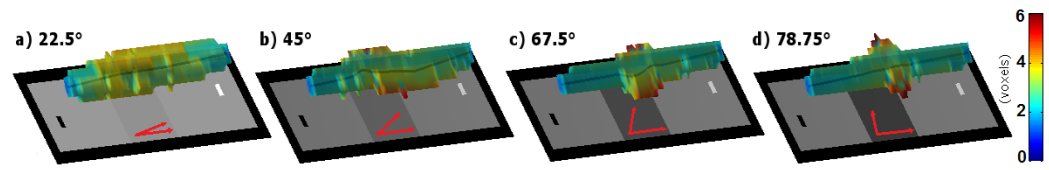

Fig. 3 Four more dMR phantom images of a single tract, instead with a narrow band of simulated crossing fibers at increasing angles. Tract curves are sampled between end points and tract confidence regions are plotted with the sample mean tract above a representative FA slice of each phantom. Each pair of red arrows indicates the primary tensor directions of the main and crossing fibers.

a noticeable bulge in the resulting confidence region. The end-points are selected to be anatomically consistent across subjects, removing the need for image registration. Fig. 4 shows that, as expected, the trajectory of the sampled tract becomes more uncertain at the region of fiber crossing. This elevated uncertainty causes a thickening of the confidence region across this area. Measurements of the confidence region thickness (diameter) in Table 1 show that the thickening is present in all subjects and that the thickness both inside and outside of this region is reasonably consistent across subjects.

Table 1 Average confidence region thickness (in mm) per section of CC tract. Note the consistently greater confidence region thickness within the crossing region, indicative of greater uncertainty.

\begin{tabular}{|c|cccccccccccc|cc|}
\hline Subject & $\mathrm{a}$ & $\mathrm{b}$ & $\mathrm{c}$ & $\mathrm{d}$ & $\mathrm{e}$ & $\mathrm{f}$ & $\mathrm{g}$ & $\mathrm{h}$ & $\mathrm{i}$ & $\mathrm{j}$ & $\mathrm{k}$ & $\mathrm{l}$ & mean & var \\
\hline Crossing Region & 2.08 & 2.73 & 1.88 & 1.89 & 2.02 & 2.01 & 1.74 & 2.65 & 2.51 & 2.16 & 2.09 & 2.40 & $\mathbf{2 . 1 8}$ & 0.15 \\
Rest of Tract & 1.84 & 2.10 & 1.65 & 1.64 & 1.61 & 1.67 & 1.55 & 2.01 & 1.60 & 1.68 & 1.72 & 1.99 & $\mathbf{1 . 7 5}$ & 0.10 \\
\hline
\end{tabular}

Finally, we compute tract confidence regions in brains of diseased human subjects. Two subjects are afflicted with MS and the other two with malignant tumors. In each of the four subjects we examined, tracts are sampled from both hemispheres of the brain with one side showing a healthy tract and the other side containing an MS lesion or tumor on the corresponding tract. A clear bulge is expected in each example where the tracts cross the lesion/tumor, indicating increased uncertainty in tract trajectory. Conversely, confidence regions for both tracts passing through healthy brain tissue should have a more uniform thickness and be narrower. Fig. 5 shows that our confidence region appraoch provides that expected result, suggesting that tract confidence regions could be useful in measuring the severity of these abnormalities.

\section{Conclusions}

We have presented here a novel method for examining uncertainty in tractography results based on the statistical examination of sample tracts between fixed endpoints. 

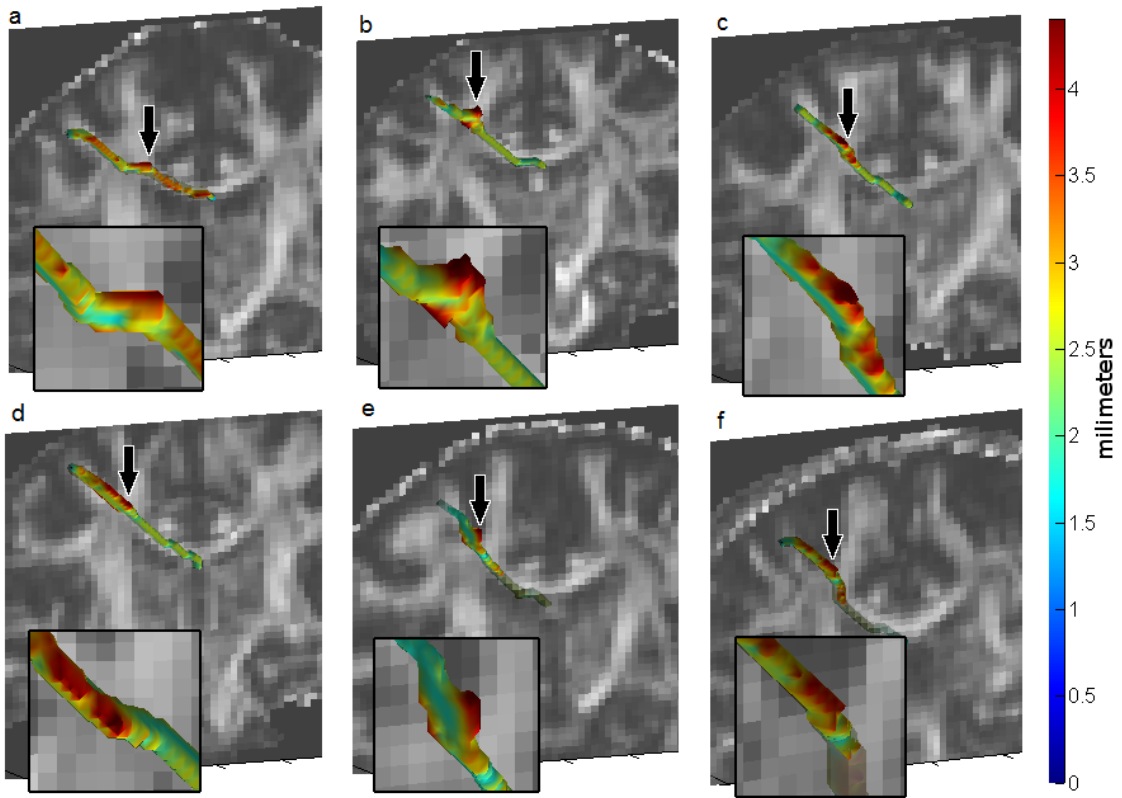

Fig. 4 Tract confidence regions along CC tracts overlaid on the subject's FA map for six subjects from the John Hopkins MILBA dataset [16]. In each example, the confidence region is thickest at the crossing between the $\mathrm{CC}$ and the corticospinal tract.

Given sample tract curves from a tractography algorithm, we compute a confidence region for the mean of the tract distribution and show how to visualize this highdimensional confidence region in the 3D image space. Finally, we showed experimentally that thickened sections in tract confidence regions correlate strongly with expected regions of high uncertainty: noisy regions, crossing fibers and regions affected by pathology. Future work will focus on examining specific features of tract confidence regions and interpreting their anatomical significance in more detail.

\section{Acknowledgements}

CJB and GH were partially supported by NSERC and BGB by IODE Canada and the Government of Alberta.

\section{References}

1. T.E.J. Behrens, M.W. Woolrich, M. Jenkinson, H. Johansen-Berg, R.G. Nunes, S. Clare, P.M. Matthews, J.M. Brady, and S.M. Smith. Characterization and propagation of uncertainty in 

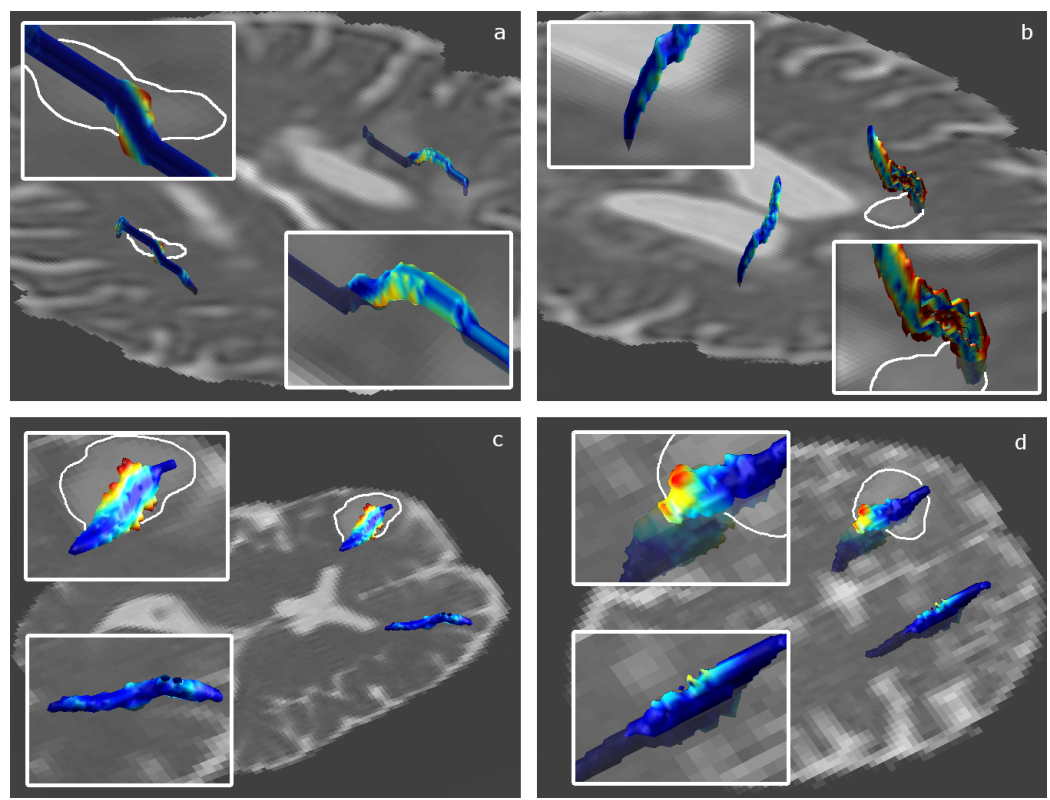

Fig. 5 Tract confidence regions for tracts in two human subjects with MS (a,b) and two subjects with brain tumors (c,d), overlaid on the subject's mean diffusivity map. Tracts are sampled in sections of the right and left superior longitudinal fasciculus in (a), splenium in (b), inferior frontooccipital fasciculus in (c) and superior fronto-occipital fasciculus in (d). In all cases, clear bulges occur as the tract passes near affected region (delineated in white).

diffusion-weighted MR imaging. Mag. Res. Med., 50(5):1077-1088, 2003.

2. S. Boyd and L. Vandenberghe. Convex Optimization. Cambridge University Press, Cambridge, UK, 2004.

3. C.J. Brown, B.G. Booth, and G. Hamarneh. k-confidence: Assessing uncertainty in tractography using k optimal paths. In Proc. of ISBI, pages 250 - 253, 2013.

4. M. Catani, R.J. Howard, S. Pajevic, and D. K. Jones. Virtual in vivo interactive dissection of white matter fasciculi in the human brain. Neuroimage, 17(1):77-94, 2002.

5. O. Ciccarelli, G.J.M. Parker, A.T. Toosy, C.A.M. Wheeler-Kingshott, G.J. Barker, P.A. Boulby, D.H. Miller, and A.J. Thompson. From diffusion tractography to quantitative white matter tract measures: a reproducibility study. Neuroimage, 18(2):348-359, 2003.

6. Olga Ciccarelli, Marco Catani, Heidi Johansen-Berg, Chris Clark, and Alan Thompson. Diffusion-based tractography in neurological disorders: concepts, applications, and future developments. The Lancet Neurology, 7(8):715-727, 2008.

7. I. Corouge, P.T. Fletcher, S. Joshi, S. Gouttard, G. Gerig, et al. Fiber tract-oriented statistics for quantitative diffusion tensor MRI analysis. Medical Image Analysis, 10(5):786-798, 2006.

8. M.-A. Côté, A. Boré, G. Girard, J.-C. Houde, and M. Descoteaux. Tractometer: Online evaluation system for tractography. In Proc. of MICCAI, pages 699-706, 2012.

9. N.R. Draper and H. Smith. Applied Regression Analysis, volume 2. John Wiley and Sons Ltd., 1981.

10. T.B. Dyrby, L.V. Sogaard, G.J. Parker, D.C. Alexander, N.M. Lind, W.F.C. Baaré, A. HaySchmidt, N. Eriksen, B. Pakkenberg, O.B. Paulson, and J. Jelsing. Validation of in vitro probabilistic tractography. NeuroImage, 37(4):1267 - 1277, 2007. 
11. O. Friman, G. Farneback, and C. F. Westin. A bayesian approach for stochastic white matter tractography. IEEE Trans. Med. Imag., 25:965-978, 2006.

12. P.E. Gill, W. Murray, and M.H. Wright. Practical Optimization. Academic Press, London, UK, 1981.

13. M. Lazar and A.L. Alexander. Bootstrap white matter tractography (boot-trac). NeuroImage, 24(2):524-532, 2005

14. D. Le Bihan, C. Poupon, A. Amadon, and F. Lethimonnier. Artifacts and pitfalls in diffusion MRI. J. Mag. Res. Imag., 24(3):478-488, 2006.

15. P.C. Mahalanobis. On the generalized distance in statistics. In Proceedings of the National Institute of Sciences of India, volume 2, pages 49-55. New Delhi, 1936.

16. S. Mori. John Hopkins Medical Institute: Laboratory of Brain Anatomical MRI, in vivo human database. http://lbam.med.jhmi.edu/, accessed February 2013.

17. L. A. Wolsey. Integer Programming. John Wiley and Sons Ltd., 1998.

18. Kei Yamada, Koji Sakai, Kentaro Akazawa, Sachiko Yuen, and Tsunehiko Nishimura. Mr tractography: a review of its clinical applications. Magnetic Resonance in Medical Sciences, 8(4):165-174, 2009. 